\title{
On the Accuracy of the Numerical Solution of the Dirichlet Problem by Finite Differences ${ }^{1}$
}

\author{
J. L. Walsh ${ }^{2}$ and David Young ${ }^{3}$
}

\begin{abstract}
This paper derives numerical bounds for the error, in certain closed regions, of the difference analog of the Dirichlet problem. It is concerned only with the difference between the exact solution of the difference equation and the solution of the Dirichlet problem. The error bounds obtained involve quantities which can actually be computed, such as the mesh size, and the oscillation and modulus of continuity of the given function on the boundary. So far as the method is concerned, the chief novelty is the use of the difference analogs of harmonic measure and the Schwarz Alternating Process.
\end{abstract}

\section{Introduction}

Although finite difference methods afford a powerful tool for obtaining numerical solutions of partial differential equations, little is known about the accuracy. It is the purpose of this paper to derive numerical bounds for the error, in certain closed regions, of the difference analog of the Dirichlet problem. We shall be concerned only with the difference between the exact solution of the difference equation and the solution of the Dirichlet problem. The error bounds that we obtain involve quantities that can actually be computed such as the mesh size, and the oscillation and modulus of continuity of the given function on the boundary. So far as method is concerned, the chief novelty is the use of the difference analogs of harmonic measure and the Schwarz Alternating Process.

Gerschgorin [4] ${ }^{4}$ derived error bounds for boundary value problems associated with elliptic partial differential equations. These, and similar bounds derived by Collatz [2] and Mikeladze [10] involve bounds, in the closed region, of certain partial derivatives of the solution of the differential equation. However, the solution of the differential equation itself is not known, to say nothing of its derivatives, (although approximate values for the derivatives may sometimes be found by examining the corresponding difference quotients). Also, it may happen that although the derivatives in question are not bounded in the closed region, the solution of the difference equation may still converge to the solution of the differential equation.

Rosenbloom [13] presented an error bound for the Dirichlet problem which is closely related to Gerschgorin's but which utilizes special properties of harmonic functions. By use of well-known inequalities giving bounds for the partial derivatives of a harmonic function at an interior point in terms of the oscillation on the boundary and the distance from the boundary, he obtains upper bounds for the derivatives in closed subregions. Then, by solving the difference equation on subregions and, as the mesh size approaches zero, letting these subregions approach the given region, Rosenbloom obtains an error bound involving $\omega^{*}(\delta)$, the modulus of continuity in the closed region of the solution of the Dirichlet problem. However, Rosenbloom does not discuss the question of finding $\omega^{*}(\delta)$ in terms of $\omega(\delta)$, the known modulus of continuity of the given function on the boundary. Furthermore, in practical numerical work one would not wish to change the boundary of the network as the mesh size is decreased.

In section 3 we use the explicit solution of the difference analog of the Dirichlet problem for the rectangle, obtained by Le Roux [9] and by Phillips and Wiener [12], to derive an error bound involving bounds for the derivatives of the given function on the boundary. Wasow [15] has obtained error bounds for the rectangle which are applicable when the boundary values

\footnotetext{
1 Presented to the American Mathematical Society, September 1951; abstract published in Bul. Am. Math. Soc. 57, 478 (1951). The paper was prepared in part while Professor Walsh was engaged as consultant on a National Bureau of Standards contract with the University of California at Los Angeles. The research was also supported by the Office of Naval Research under Contracts N5ori-07634 and N5ori-76, Project 22, with Harvard University, and later by the Office of Ordnance Research, U. S. Army, under Contract DA-36 034-ORD 966.

2 Harvard University; consultant, National Bureau of Standards contract with the University of California at Los Angeles.

University of Maryland.

4 Figures in brackets indicate the literature references at the end of this paper.
} 
have bounded third derivatives. Our results are somewhat more general in that we allow the second and third derivatives to fail to exist at a finite number of points. Also, we obtain error bounds for the case where only the second derivative is bounded, and we allow the first and second derivatives to fail to exist at a finite number of points. In sections 4 and 5 harmonic measure and its finite difference analog, discrete harmonic measure, are used to obtain bounds for the moduli of continuity of harmonic and discrete harmonic functions, respectively, in certain regions in terms of $\omega(\delta)$. Phillips and Wiener [12] showed the existence of such bounds, whereas we obtain suitable bounds in a precise numerical form. Then, in section 6 these upper bounds are used to yield a uniform error estimate for the closed rectangle in terms of the oscilliation on the boundary and $\delta(\omega)$. In section 7 the extension to regions made up of two or more overlapping rectangles is discussed.

\section{Discrete Harmonic Functions}

Let $h$ and $k$ be arbitrary positive numbers, and let $L[h, k]$ denote the set of points $(x, y)$ such that both $x / h$ and $y / k$ are integers. Let $\Omega$ be a simply connected closed region with interior $R$ such that the boundary $S$ of $\Omega$ consists of straight lines, each of which is parallel to a coordinate axis and contains a point of $L[h, k]$. Let $\Omega_{L}$ denote the subset of points of $L[h, k]$ contained in $\Omega$. Two points $\left(x_{1}, y_{1}\right)$ and $\left(x_{2}, y_{2}\right)$ of $L[h, k]$ are adjacent if

$$
\left[\left(x_{1}-x_{2}\right) / h\right]^{2}+\left[\left(y_{1}-y_{2}\right) / k\right]^{2}=1 \text {. }
$$

A point of $\Omega_{L}$ is an interior point of $\Omega_{L}$ if the four adjacent points belong to $\Omega_{L}$. All other points of $\Omega_{L}$ are boundary points. We let $R_{L}$ and $S_{L}$ denote, respectively, the set of interior and boundary points of $\Omega_{L}$. Evidently, we have $R_{L} \subseteq R$ and $S_{L} \subseteq S$.

A function $U(x, y)$ defined on $\Omega_{L}$ is said to be discrete harmonic, ${ }^{5}$ (d. h.) in $R_{L}$ if it satisfies the difference equation

$$
\begin{aligned}
\delta^{2}[U(x, y)]=\left[2 \sigma^{2} /\left(1+\sigma^{2}\right)\right][U(x+h, y)+ & U(x-h, y)-2 U(x, y)] \\
& +\left[2 /\left(1+\sigma^{2}\right)\right][U(x, y+k)+U(x, y-k)-2 U(x, y)]=0,
\end{aligned}
$$

where

$$
\sigma=k / h \text {. }
$$

The finite difference analog of the Dirichlet problem is the following problem: given a function $f(x, y)$ defined on $S$, to find a function $U(x, y)$ defined on $\Omega_{L}$, d.h. in $R_{L}$ and coinciding with $f(x, y)$ on $S_{L}$. The existence and uniqueness of a solution of this problem for bounded regions is easy to prove; see for instance Gerschgorin [4]. The convergence to the solution of the Dirichlet problem has been proved using nonconstructive methods by Le Roux [9], Phillips and Wiener [12], and others.

\section{Error Estimate for the Rectangle Under Differentiability Assumptions}

Let $\Omega$ be bounded by the lines $x=0, x=a, y=0$, and $y=b$, where $a=A h, b=B k$ and $A$ and $B$ are positive integers. Let $f(x, y)$ be defined and continuous on $S$ and let $u(x, y)$ and $U(x, y)$ denote respectively the solution of the Dirichlet problem and its finite difference analog with boundary values determined by $f(x, y)$. In this section we shall derive an upper bound for the error $U(x, y)-u(x, y)$ in the region $\Omega_{L}$, under certain assumptions about the derivatives of $f(x, y)$. If $f_{i}(x, y)$ is defined and continuous on $S$, we denote generically by $u_{i}(x, y)$ and $U_{i}(x, y)$ the solutions respectively of the Dirichlet problem and its finite difference analog with boundary values $f_{1}(x, y)$.

First, one can verify directly that the function $u_{1}(x, y)$ defined by

$$
\begin{aligned}
u_{1}(x, y)=f(0,0)+[f(a, 0)-f(0,0)](x / a)+[f(0, b) & -f(0,0)](y / b) \\
& +[f(a, b)+f(0,0)-f(a, 0)-f(0, b)](x / a)(y / b),
\end{aligned}
$$

Heilbronn [5] introduced the term discrete harmonic function, and studied the properties of these functions. 
which is linear on every line parallel to either coordinate axis, is both harmonic in $R$ and d.h. in $R_{L}$. Thus if $f_{1}(x, y)$ is defined on $S$ and equal to $u_{1}(x, y)$ on $S$, then $u_{1}(x, y)=U_{1}(x, y)$ in $\Omega_{L}$.

Next, if $f_{2}(x, y)=f(x, y)-f_{1}(x, y)$ on $\mathrm{S}$, then we have by linearity of Laplace's equation and $(3.1)$

$$
\begin{aligned}
U(x, y)-u(x, y) & =\left[U_{2}(x, y)+U_{1}(x, y)\right]-\left[u_{2}(x, y)+u_{1}(x, y)\right] \\
& =U_{2}(x, y)-u_{2}(x, y) .
\end{aligned}
$$

Evidently both $U_{2}(x, y)$ and $u_{2}(x, y)$ vanish at all the corners of $\Omega$.

We study arbitrary boundary values $f(x, y)$ by studying in turn four functions each of which has the boundary values $f_{2}(x, y)$ on one side of $\Omega$ and values identically zero on the other three sides. By the symmetry of the situation, it is sufficient to study in detail only one of the latter functions. Let us set $f_{3}(x, y)=f_{2}(x, 0)$ when $y=0$ and $f_{3}(x, y)=0$ elsewhere on $S$. Since $f_{2}(x, y)$ is continuous and vanishes at the corners of $\Omega, f_{3}(x, y)$ is continuous on $S$.

It can be verified directly ${ }^{6}$ that if the Fourier series of $f_{3}(x, 0)$ converges to $f_{3}(x, 0)$, then we have

$$
u_{3}(x, y)=\sum_{n=1}^{\infty} A_{n} \sin (n \pi x / a) \frac{\sinh [(n \pi b / a)(1-y / b)]}{\sinh (n \pi b / a)}
$$

where

$$
A_{n}=(2 / a) \int_{0}^{a} f_{3}(t, 0) \sin (n \pi t / a) d t
$$

and that ${ }^{7}$

$$
U_{3}(x, y)=\sum_{n=1}^{A} A_{n}^{*} \sin (n \pi x / a) \frac{\sinh [(m \pi b / a)(1-y / b)]}{\sinh (m \pi b / a)},
$$

where

$$
A_{n}^{*}=(2 h / a) \sum_{j=1}^{A-1} \sin (n \pi j h / a) f_{3}(j h, 0)
$$

and where $m$ and $n$ satisfy the relation

$$
\sinh (m \pi k / 2 a)=\sigma \sin (n \pi h / 2 a) .
$$

Now let $g(x) \equiv f_{3}(x, 0)$. We now prove the following theorem:

Theorem 3.1 Let $g(x)$ satisfy the following conditions: (a) $g(x)$ and its first $(s-2)$ derivatives are continuous, $0 \leq x \leq a$; (b) $g^{(s-1)}(x)$ fails to exist or fails to be continuous for, at most, a finite number of points in the interval $0 \leq x \leq a$, and $g^{(s-1)}(x)$ is uniformly continuous in each open interval in which it exists and is continuous; (c) $g^{(s)}(x)$ exists, except possibly for a finite number of points, and is bounded, $0 \leq x \leq a$.

Then uniformly for all $(x, y) \epsilon \Omega_{L}$, we have

if $s=2$, and

$$
\left|U_{3}(x, y)-u_{3}(x, y)\right| \leq(2+C) D_{2}(h / a),
$$

if $s=3$, where

$$
\left|U_{3}(x, y)-u_{3}(x, y)\right| \leq(1+C e) D_{3}(h / a)^{2},
$$

$$
\begin{gathered}
C=(1 / 24) \pi^{2}\left(1+\sigma^{2}\right) \operatorname{coth}(\alpha \pi b / a) / \alpha e \\
D_{2}=2(a / \pi)^{2}\left[2 J_{1} M_{1} / a+M_{2}\right], \quad D_{3}=2(a / \pi)^{3}\left[2\left(J_{2}+1\right) M_{2} / a+M_{3}\right] \\
\alpha=(2 / \pi \sigma) \sinh ^{-1}(\sigma),
\end{gathered}
$$

- See, for instance [8], pages 95 and 96 .

${ }^{7}$ See, for instance, Le Roux [9] or Phillips and Wiener [12]. 
and $J_{i}, i=1,2$, denotes the number of points in the open interval $0<x<a$ at which $g^{(i)}(x)$ does not exist or is not continuous. Here $M_{i}, i=1,2,3$, denotes the least upper bound of the modulus of $g^{(i)}(x)$.

Proof.

Lemma 3.1. If $m$ and $n$ satisfy (3.7), then

$$
0 \leq n-m \leq(1 / 24)\left(1+\sigma^{2}\right) \pi^{2} n^{3}(h / a)^{2}, \quad(n=1,2, \ldots, A) .
$$

Proof. Let $p=m \pi k / 2 a, \quad q=n \pi h / 2 a$. Then (3.7) takes the form

$$
\sinh p=\sigma \sin q .
$$

We now study the function $p(q)$ defined by (3.14) in the interval $0 \leq q \leq \pi / 2$. One can verify directly that $p(0)=0, p^{\prime}(0)=\sigma$, and

$$
\begin{gathered}
p^{\prime \prime}(q)=\frac{-\sigma\left(1+\sigma^{2}\right) \sin q}{\left(1+\sigma^{2} \sin ^{2} q\right)^{3 / 2}} \leq 0, \\
p^{\prime \prime \prime}(q)=\frac{-\sigma\left(1+\sigma^{2}\right)\left(1-2 \sigma^{2} \sin ^{2} q\right) \cos q}{\left(1+\sigma^{2} \sin ^{2} q\right)^{5 / 2}},
\end{gathered}
$$

where primes denote differentiation with respect to $q$. By the extended mean value theorem, since $p^{\prime \prime}(q) \leq 0$, we have $p \leq \sigma q$. Therefore, $m \leq n$. On the other hand, we also have

$$
\left|p^{\prime \prime \prime}(q)\right| \leq \frac{\sigma\left(1+\sigma^{2}\right)\left|1-2 \sigma^{2} \sin ^{2} q\right|}{\left(1+\sigma^{2} \sin ^{2} q\right)^{5 / 2}} \leq \sigma\left(1+\sigma^{2}\right)
$$

since $\left|\left(1-2 \sigma^{2} \sin ^{2} q\right)\left(1+\sigma^{2} \sin ^{2} q\right)^{-5 / 2}\right| \leq 1$.

Again using the extended mean value theorem, we get

and

$$
p(q)-\sigma q=(1 / 6) q^{3} p^{\prime \prime \prime}(\xi) \quad(0<\xi<q)
$$

and the lemma follows.

$$
|p(q)-\sigma q| \leq(1 / 6) q^{3} \sigma\left(1+\sigma^{2}\right)
$$

Lemma 3.2. If $m$ and $n$ satisfy (3.7), and if $\alpha$ is given by (3.12), then

$$
m \geq \alpha n, \quad(n=1,2, \ldots, A) .
$$

Proof. Defining $p$ and $q$ by (3.14), we have

$$
\frac{d}{d q}(p(q) / q)=\left[p^{\prime}(q) q-p(q)\right] / q^{2}
$$

By the mean value theorem, we have $p(q)=q p^{\prime}(\xi) \quad(0<\xi<q)$. Since, as shown in the proof of lemma 3.1, $p^{\prime \prime}(q) \leq 0$, we have $p^{\prime}(q) \leq p^{\prime}(\xi)$ and $p^{\prime}(q) q-p(q) \leq 0$. Therefore, the ratio $p(q) / q$ is a nonincreasing function of $q,(0 \leq q \leq \pi / 2)$. Its minimum value in the interval $0 \leq q \leq \pi / 2$ is assumed when $q=\pi / 2$. Therefore, $p(q) / q \geq p(\pi / 2) /(\pi / 2)=(2 / \pi) \sinh ^{-1}(\sigma)$, and the lemma follows.

Now for convenience, let us define the function

$$
\Gamma_{m}(y)=\frac{\sinh [(m \pi b / a)(1-y / b)]}{\sinh (m \pi b / a)} .
$$

We shall study its behavior as a function of both $m$ and $y$, where $m$ is assumed to be a continuous variable.

Lemma 3.3. If $m \geq 0$, then for $0 \leq y \leq b$ we have

$$
0 \geq \frac{d}{d m} \Gamma_{m}(y) \geq-(\pi y / a) \operatorname{coth}(m \pi b / a) \exp [-m \pi y / a]
$$


Proof. Differentiating (3.16) with respect to $m$ we obtain

$$
\begin{aligned}
\frac{d}{d m} \Gamma_{m}(y) & =(\pi b / a)\{(1-y / b) \cosh [(m \pi b / a)(1-y / b)] \sinh (m \pi b / a) \\
& -\cosh (m \pi b / a) \sinh [(m \pi b / a)(1-y / b)]\} \sinh ^{-2}(m \pi b / a) \\
= & (\pi b / a) \Gamma_{m}(y)\{(1-y / b) \operatorname{coth}[(m \pi b / a)(1-y / b)]-\operatorname{coth}(m \pi b / a)\} .
\end{aligned}
$$

We note that for $K>0, x>0$,

$$
\frac{d}{d x}(x \operatorname{coth}(K x))=\operatorname{coth}(K x)-K x \operatorname{csch}^{2}(K x)=\frac{1}{2} \frac{\sinh (2 K x)-2 K x}{\sinh ^{2}(K x)} .
$$

But since $\sinh (2 K x) \geq 2 K x$, the last expression is nonnegative; therefore by the mean value theorem we have

$$
(1-y / b) \operatorname{coth}[(m \pi b / a)(1-y / b)] \leq \operatorname{coth}(m \pi b / a), \quad(0 \leq y \leq b),
$$

and

$$
\frac{d}{d m} \Gamma_{m}(y) \leq 0
$$

On the other hand,

$$
\begin{aligned}
\frac{d}{d m} \Gamma_{m}(y) & =(\pi b / a)\left\{(1-y / b) \frac{\cosh [(m \pi b / a)(1-y / b)]}{\sinh (m \pi b / a)}-\operatorname{coth}(m \pi b / a) \Gamma_{m}(y)\right\} \\
& =(\pi b / a) \operatorname{coth}(m \pi b / a)\left\{(1-y / b) \frac{\cosh [(m \pi b / a)(1-y / b)]}{\cosh (m \pi b / a)}-\Gamma_{m}(y)\right\} \\
& =(\pi b / a) \operatorname{coth}(m \pi b / a)\left\{(1-y / b) \frac{\sinh (m \pi y / a)}{\sinh (m \pi b / a) \cosh (m \pi b / a)}-(y / b) \Gamma_{m}(y)\right\}
\end{aligned}
$$

For $0 \leq y \leq b$, the terms in the brackets are, respectively, nonnegative and nonpositive, whence

$$
\frac{d}{d m} \Gamma_{m}(y) \geq-(\pi y / a) \operatorname{coth}(m \pi b / a) \Gamma_{m}(y) .
$$

Finally, we note that

$$
\Gamma_{m}(y)=\exp (-m \pi y / a) \frac{1-\exp [-2(m \pi b / a)(1-y / b)]}{1-\exp [-2 m \pi b / a]} \leq \exp (-m \pi y / a),
$$

provided $^{8} 0 \leq y \leq b$. The lemma now follows.

LEMMa 3.4. If $m$ and $n$ satisfy (3.7), then

$$
\left|\left[\frac{d}{d m} \Gamma_{m}(y)\right]_{m=m_{1}}\right| \leq[\operatorname{coth}(\alpha \pi b / a) / \alpha e] n^{-1}, \quad(1 \leq n \leq A), \quad\left(m \leq m_{1} \leq n,\right)
$$

where $\alpha$ is given by (3.12).

Proof. It is easily verified that if $0 \leq t, 0 \leq K$, then $\left|t e^{-K t}\right| \leq(K e)^{-1}$. Therefore, $y \exp (-m \pi y / a) \leq[(m \pi / a) e]^{-1}$. The lemma follows from lemmas 3.2 and 3.3, and from the fact that $\operatorname{coth}(x)$ is a decreasing function of $x$, for $x \geq 0$.

Lemma 3.5. If $m$ and $n$ satisfy (3.7), then

$$
\left|\Gamma_{m}(y)-\Gamma_{n}(y)\right| \leq C(h / a)^{2} n^{2}, \quad(0 \leq y \leq b), \quad(1 \leq n \leq A),
$$

where $C$ and $\alpha$ are given by (3.10) and (3.12), respectively.

${ }^{8}$ This inequality was proved by Phillips and Wiener [12]. 
Proof. By the mean value theorem we have

$$
\left|\Gamma_{m}(y)-\Gamma_{n}(y)\right| \leq \operatorname{Max}_{m \leq m_{1} \leq n}\left|\left[\frac{d}{d m} \Gamma_{m}(y)\right]_{m=m_{1}}\right| \cdot|m-n| .
$$

The lemma follows from (3.13) and (3.17).

Lemma 3.6. If $A_{n}$ is defined by (3.4), then

$$
\left|A_{n}\right| \leq D_{s} / n^{s}, \quad(n=2,3),
$$

where $D_{s}$ is defined by (3.11).

Proof. The proof involves the use of repeated integration by parts of the integral expression for $A_{n}$, and is similar to that given by Jackson[8, p. 13-14] for the case $s=2$. We omit the details.

We now define the function $f_{4}(x, y)$ :

$$
f_{4}(x, y)=0 \text { unless } y=0 \quad \text { and } \quad f_{4}(x, 0)=\sum_{n=1}^{A} A_{n} \sin (n \pi x / a) .
$$

For $s=2,3$ and $0 \leq x \leq a$, we have

$$
\left|f_{4}(x, 0)-g(x)\right|=\left|\sum_{n=A+1}^{\infty} A_{n} \sin (n \pi x / a)\right| \leq D_{s} \sum_{n=A+1}^{\infty} n^{-s} \leq D_{s} \int_{A}^{\infty} t^{-s} d t=D_{s} A^{1-s} /(s-1),
$$

or

$$
\left|f_{4}(x, 0)-g(x)\right| \leq\left[D_{s} /(s-1)\right](h / a)^{s-1} .
$$

On the other hand, if we replace $f_{3}(x, 0)$ by $f_{4}(x, 0)$ in (3.4) and (3.6), we get $A_{n}^{*}=A_{n}$, $(n=1,2, \ldots, A-1)$. Therefore, for $(x, y) \in L[h, k]$, we have, since $\sin (A \pi x / a)=0$,

$$
U_{4}(x, y)-u_{4}(x, y)=\sum_{n=1}^{A-1} A_{n} \sin (n \pi x / a)\left[\Gamma_{m}(y)-\Gamma_{n}(y)\right]
$$

and

$$
\left|U_{4}(x, y)-u_{4}(x, y)\right| \leq \sum_{n=1}^{A-1}\left|A_{n}\right| \cdot\left|\Gamma_{m}(y)-\Gamma_{n}(y)\right| \leq C D_{s}(h / a)^{2} \sum_{n=1}^{A-1} n^{2-s}
$$

by lemma 3.5 and (3.18). It can be shown that

$$
\sum_{n=1}^{A-1} n^{2-s} \leq 1+\int_{1}^{A} t^{2-s} d t \leq\left\{\begin{array}{c}
A=(h / a)^{-1}, \quad(s=2) \\
1+\log A=1-\log (h / a), \quad(s=3) .
\end{array}\right.
$$

Therefore, if $s=2$,

$$
\left|U_{4}(x, y)-u_{4}(x, y)\right| \leq C D_{2}(h / a)
$$

Evidently $(3,22)$ could be used to obtain an error bound for the case $s=3$, and this was done in the original manuscript of the present paper. Since then, however, a paper by Wasow [15] has appeared which contains a stronger result for the case $s=3$ than the original form of theorem 3.1. A combination of his methods and the original ones now yields an improvement (the present theorem 3.1) of Wasow's result in this case since we allow $g^{(3)}(x)$ and $g^{(2)}(x)$ to fail to exist for a finite number of points.

Thus following [15], we obtain from lemmas 3.1, 3.2, 3.3, and the mean value theorem

$$
\left|\Gamma_{m}(y)-\Gamma_{n}(y)\right| \leq C \alpha e(h / a)^{2}(\pi y / a) n^{3} \exp (-\alpha n \pi y / a) .
$$


By (3.20) and lemma 3.6 we have

$$
\left|U_{4}(x, y)-u_{4}(x, y)\right| \leq C D_{3} \alpha e(h / a)^{2}(\pi y / a) \sum_{n=1}^{\infty} \exp (-\alpha n \pi y / a) \leq C e D_{3}(h / a)^{2},
$$

since for $y>0, \sum_{n=1}^{\infty} \exp (-\alpha n \pi y / a)=[\exp (\alpha \pi y / a)-1]^{-1} \leq(\alpha \pi y / a)^{-1}$.

Since d.h. functions possess mean value properties, they assume their maximum and minimum values on the boundary as do harmonic functions. Since the functions $\left[u_{3}(x, y)-u_{4}(x, y)\right]$ and $\left[U_{3}(x, y)-U_{4}(x, y)\right]$ are harmonic and d.h., respectively, we have

$$
\left|u_{3}(x, y)-u_{4}(x, y)\right| \leq \operatorname{Max}_{0 \leq x \leq a}\left|g(x)-f_{4}(x, 0)\right|, \quad\left|U_{3}(x, y)-U_{4}(x, y)\right| \leq \operatorname{Max}_{0 \leq x \leq a}\left|g(x)-f_{4}(x, 0)\right| .
$$

Therefore, in $\Omega_{L}$

$$
\begin{aligned}
\left|U_{3}(x, y)-u_{3}(x, y)\right| & \leq\left|U_{3}(x, y)-U_{4}(x, y)\right|+\left|U_{4}(x, y)-u_{4}(x, y)\right|+\left|u_{4}(x, y)-u_{3}(x, y)\right| \\
& \leq\left|U_{4}(x, y)-u_{4}(x, y)\right|+2 \underset{0 \leq x \leq a}{\operatorname{Max}}\left|g(x)-f_{4}(x, 0)\right| .
\end{aligned}
$$

By (3.19), (3.23), and (3.24), the theorem follows.

\section{Harmonic Measure}

Let $\Omega$ denote a simply connected region with interior $R$ and boundary $S$. Let $S^{\prime}$ denote a subset of $S$ consisting of a finite number of connected subsets of $S$. We define the harmonic measure, (h.m.), $H\left[(x, y), S^{\prime}, \Omega\right]$ as the unique function which is harmonic and bounded in $R$, is continuous in $\Omega$ except perhaps at a finite number of points of $S$, and equals unity on $S^{\prime}$ and zero on $S-S^{\prime}$. The properties of harmonic measure have been studied in considerable detail, see Nevanlinna [11], chapter III.

By analogy we define discrete harmonic measure, (d.h.m.), for regions of the type described in section 2, as follows: $H_{L}\left[(x, y), S^{\prime}, \Omega_{L}\right]$ is a function d.h. and bounded in $R_{L}$, equal to unity on $S_{L} \cap S^{\prime}$ and to zero on $\left(S-S^{\prime}\right) \cap S_{L}$.

For bounded regions the existence and uniqueness of h.m. is well known, see [11]. The existence and uniqueness for the half plane can be proved by the use of conformal mapping.

The existence and uniqueness of d.h.m. for bounded regions follows from the existence and uniqueness of the solution of the difference analog of the Dirichlet problem. Later we shall prove existence and uniqueness of d.h.m. for a half plane and for certain other unbounded regions.

We list some elementary properties of d.h.m., which are analogs of well-known properties of h.m.

If $S^{\prime} \subseteq S^{\prime \prime} \subseteq S$, then

$$
0 \leq H_{L}\left[(x, y), S^{\prime}, \Omega_{L}\right] \leq 1 \text { for all } S^{\prime} \subseteq S .
$$

$$
H_{L}\left[(x, y), S^{\prime}, \Omega_{L}\right] \leq H_{L}\left[(x, y), S^{\prime \prime}, \Omega_{L}\right]
$$

If $S^{\prime}$ is included in the boundary of both $\Omega_{L}$ and $\Omega_{L}^{*}$ where $\Omega_{L} \subset \Omega_{L}^{*}$ and if $(x, y) \epsilon \Omega_{L}$, then

$$
H_{L}\left[(x, y), S^{\prime}, \Omega_{L}\right] \leq H_{L}\left[(x, y), S^{\prime}, \Omega_{L}^{*}\right]
$$

The first property follows at once from the fact that the maximum and minimum values of d.h. functions are assumed on the boundary. The second follows from this fact and from the fact that the expression $\left\{H_{L}\left[(x, y), S^{\prime \prime}, \Omega_{L}\right]-H_{L}\left[(x, y), S^{\prime}, \Omega_{L}\right]\right\}$ is nonnegative on $S_{L}$. The third follows since by the maximum and minimum principles $H_{L}\left[(x, y), S^{\prime}, \Omega_{L}^{*}\right] \geq 0$ and hence $\left\{H_{L}\left[(x, y), S^{\prime}, \Omega_{L}^{*}\right]-H_{L}\left[(x, y), S^{\prime}, \Omega_{L}\right]\right\} \geq 0$ for any point $(x, y)$ on the boundary of $\Omega_{L}$. This is the so-called principle of gebietserweiterung.

We shall use h.m. and d.h.m. for two purposes. In this section and in section 5 lower bounds will be derived to enable us to obtain upper bounds for the modulus of continuity of harmonic and d.h. functions in a closed region in terms of their moduli of continuity on the 
boundary. In section 7, upper bounds will be derived which will enable us to use the Schwarz Alternating Process and its difference analog for overlapping rectangles.

Let $\Omega$ denote the rectangle of section 3 , and let $S^{\prime}$ denote the side contained in the line $y=0$.

Theorem 4.1. If $(x-a / 2)^{2}+y^{2} \leq \delta^{2}$, then

where

$$
\begin{gathered}
H\left[(x, y), S^{\prime}, \Omega\right] \geq 1-\nu(b / a)(\delta / a), \\
H_{L}\left[(x, y), S^{\prime}, \Omega_{L}\right] \geq 1-\nu(b / a)(\delta / a),
\end{gathered}
$$

Proof. Let $u(x, y), U(x, y)$ be harmonic and d.h. functions respectively in $R$ vanishing on $S-S^{\prime}$ and equal to $\sin (\pi x / a)$ on $S^{\prime}$. By (3.3) to (3.7) we have

$$
u(x, y)=\sin (\pi x / a) \frac{\sinh [(\pi b / a)(1-y / b)]}{\sinh (\pi b / a)}, \quad \text { and } \quad U(x, y)=\sin (\pi x / a) \frac{\sinh [(m \pi b / a)(1-y / b)]}{\sinh (m \pi b / a)},
$$

where $\sinh (m \pi k / 2 a)=\sigma \sin (\pi h / 2 a)$.

By lemma 3.1 we have $m \leq 1$. Also by lemma 3.3 we have $(d / d m) \Gamma_{m}(y) \leq 0$. Therefore, $\Gamma_{m}(y) \geq \Gamma_{1}(y)$, and we get

$$
U(x, y) \geq u(x, y)
$$

Now, for $0 \leq \theta \leq \pi / 2$ we have ${ }^{9} \sin \theta \geq(2 / \pi) \theta$; and hence for $0 \leq x \leq a / 2$, we have $\sin (\pi x / a) \geq 2 x / a=1-(2 / a)(a / 2-x)$. But since $\sin [(\pi / a)(a / 2+\rho)]=\sin [(\pi / a)(a / 2-\rho)]$ for all $\rho$ it follows that $\sin (\pi x / a) \geq 1-(2 / a)|a / 2-x|,(0 \leq x \leq a)$.

Also

$$
\left|\frac{d}{d y} \sinh [(\pi b / a)(1-y / b)]\right|=|(\pi / a) \cosh [(\pi b / a)(1-y / b)]| \leq(\pi / a) \cosh (\pi b / a),
$$

and by the mean value theorem

Therefore,

$$
\sinh [(\pi b / a)(1-y / b)] \geq \sinh (\pi b / a)-(\pi y / a) \cosh (\pi b / a) .
$$

$$
1-u(x, y) \leq 1-\{[1-(2 / a)|a / 2-x|][1-(\pi y / a) \operatorname{coth}(\pi b / a)]\} \leq(2 / a)|a / 2-x|+(\pi y / a) \operatorname{coth}(\pi b / a) .
$$

By the Schwarz inequality the last expression does not exceed

$$
(1 / a)\left[(a / 2-x)^{2}+y^{2}\right]^{1 / 2} \nu(b / a) \leq(\delta / a) \nu(b / a),
$$

provided $(x-a / 2)^{2}+y^{2} \leq \delta^{2}$.

We now observe that $H\left[(x, y), S^{\prime}, \Omega\right] \geq u(x, y), \quad H_{L}\left[(x, y), S^{\prime}, \Omega_{L}\right] \geq U(x, y)$. The theorem now follows from (4.7).

Now let $\Omega_{L}$ denote the subset of $L[h, k]$ such that $y \geq 0$ and let $R_{L}$ and $S_{L}$ denote the interior and boundary of $\Omega_{L}$, respectively.

THeOREM 4.2. For the region $\Omega_{L}$, d.h.m. exists and is unique.

Proof. Consider the sequence $\left\{H_{L}^{(n)}(x, y)\right\}$, where

$$
H_{L}^{(n)}(x, y)=H_{L}\left[(x, y), S^{\prime} \cap S_{L}^{(n)}, \Omega_{L}^{(n)}\right],
$$

and where $\Omega_{L}^{(n)}$ denotes the rectangle with vertices $(n h, 0),(-n h, 0),(n h, n k)$ and $(-n h, n k)$. Evidently $H_{L}^{(n)}(x, y)$ exists, since d.h.m. exists for bounded regions. By (4.3) it follows that the sequence $\left\{H_{L}^{(n)}(x, y)\right\}$ is nondecreasing and bounded above by unity. Therefore, a unique limit exists which we denote by $H_{L}(x, y)$.

'This is Jordan's Inequality; see Copson [3, p. 136]. 
Since $H_{L}^{(n)}(x, y)$ is d.h., it satisfies (2.2). Taking limits of both sides of (2.2), we find that $H_{L}(x, y)$ satisfies $(2.2)$ and is therefore d.h. Evidently $H_{L}(x, y) \leq 1, H_{L}(x, y)=1$ on $S^{\prime} \cap S_{L}$ and $H_{L}(x, y)=0$ on $S^{\prime}-S_{L}$. Hence $H_{L}(x, y)$ has all the properties required for d.h.m.

In order to establish uniqueness, we prove

Lemma 4.1. If $U(x, y)$ is d.h., bounded in $R_{L}$ and vanishes on $S_{L}$, then $U(x, y)$ vanishes in $R_{L}$.

Proof. By hypothesis there exists a constant $P$ such that for $(x, y) \epsilon \Omega_{L}$, we have $|U(x, y)| \leq P$. Given any $(x, y) \epsilon \Omega_{L}$ and any $\epsilon>0$, let $a$ and $b$ be chosen so that $(a, b) \epsilon L[h, k]$ and

$$
a \geq P\left(x^{2}+y^{2}\right)^{1 / 2} \nu(1 / 2) / 2 \epsilon, \quad b \geq a .
$$

Let $\Omega *$ denote the rectangle with vertices at $(a, b),(-a, b),(a, 0)$, and $(-a, 0)$, and let $I_{a}$ denote the interval $(-a<x<a)$. Since $|U(x, y)| \leq P$ on $S^{*}$ and vanishes on $I_{a}$, we have

$$
|U(x, y)| \leq P H_{L}\left[(x, y), S^{*}-I_{a}, S_{L}^{*}\right] .
$$

By theorem 4.1 we have, replacing $a$ by $2 a$,

$$
H_{L}\left[(x, y), I_{a}, \Omega_{L}^{*}\right] \geq 1-\nu(b / 2 a)(\delta / 2 a),
$$

provided $x^{2}+y^{2} \leq \delta^{2}$. But by hypothesis, we have $\left(x^{2}+y^{2}\right)^{1 / 2} \leq 2 \epsilon a / P \nu(1 / 2)$. Substituting, we get

$$
H_{L}\left[(x, y), I_{a}, \Omega_{L}^{*}\right] \geq 1-\nu(b / 2 a) \epsilon / P \nu(1 / 2) .
$$

Since $b \geq a$, we have $\nu(b / 2 a) \leq \nu(1 / 2)$; hence,

$$
H_{L}\left[(x, y), I_{a}, \Omega_{L}^{*}\right] \geq 1-\epsilon / P, \quad \text { and } \quad|U(x, y)| \leq \epsilon .
$$

This proves the lemma.

The uniqueness can now be proved by assuming two d.h.m.'s and showing that their difference vanishes identically.

The proof of theorem 4.2 is complete.

We note that the existence of a unique bounded solution of the difference analog of the Dirichlet problem for $\Omega_{L}$ with bounded boundary values is almost immediate. If the boundary values are determined by $g(x)$, then the limit of the absolutely and uniformly convergent series $\sum_{\mu=-\infty}^{\infty} H_{L}\left[(x, y), \mu h, \Omega_{L}\right] g(\mu h)$ is d.h. in $R_{L}$, bounded in $\Omega_{L}$ and equals $g(x)$ on $S_{L}$. Thus a solution exists. The uniqueness follows at once from lemma 4.1.

We next consider the region $x \geq 0, y \geq 0$. To find d.h.m. for subsets of the line $y=0$ we perform a sign-changing reflection about the line $x=0$ and use theorem 4.2. Since the d.h.m. is zero on the line $x=0$, this can be done in such a way that the new function will be d.h. in $R_{L}$. Similarly to find d.h.m. for subsets of the line $y=0$ we reflect in the line $x=0$.

By similar methods the existence and uniqueness of d.h.m. can be established for other regions such as the semi-infinite strip $0 \leq x \leq a, y \geq 0$.

\section{Modulus of Continuity}

The modulus of continuity of a function $f(x, y)$ in a closed region $\Omega$ is defined by

$$
\omega(\delta)=L U B\left|f\left(x_{1}, y_{1}\right)-f\left(x_{2}, y_{2}\right)\right|,
$$

where $\left(x_{1}, y_{1}\right),\left(x_{2}, y_{2}\right) \in \Omega$ and $\left(x_{1}-x_{2}\right)^{2}+\left(y_{1}-y_{2}\right)^{2} \leq \delta^{2}$. Evidently if $\delta$ is not less than the diameter of $\Omega$ then $\omega(\delta)$ equals the oscillation of $f(x, y)$ in $\Omega$.

For harmonic functions we prove the following theorem:

Theorem 5.1. Let $\Omega$ denote a bounded simply connected closed region with interior $R$ and whose boundary $S$ is a closed Jordan curve with the following property: there exist constants $r_{0}>0$ and $\theta \geq 0$ such that for any point $P$ of $S$ there exists a circular sector with vertex at $P$, with 
radius $r_{0}$ and included angle $\theta$ containing no point of $R$. Let $u(x, y)$ be harmonic in $R$, continuous in $\Omega$ with modulus of continuity $\omega(\delta)$ on $S$. If for some positive number $D$ we have

$$
\delta \leq \operatorname{Max}\left\{r_{0}, D, D\left(r_{0} / D\right)^{(\pi+\psi) / \pi}\right\}
$$

then the modulus of continuity of $u(x, y)$ in $\Omega$ satisfies the inequality

$$
\omega^{*}(\delta) \leq \omega\left[D(\delta / D)^{\pi /(\pi+\psi)}\right]+(4 M / \pi)(\delta / D)^{\pi /(\pi+\psi)},
$$

where $\psi=\operatorname{Max}(2 \pi-\theta, \pi)$ and

$$
M=\underset{(x, y)_{\epsilon S}}{\operatorname{Max}}[u(x, y)]-\underset{(x, y) \epsilon S}{\operatorname{Min}}[u(x, y)]
$$

is the oscillation of $u(x, y)$ on $S$.

Proof.

Lemma 5.1. Let $I_{r}$ denote the interval $-r \leq x \leq r, y=0$ and let $C_{\rho}$ denote the region $x^{2}+y^{2} \leq \rho^{2}, y \geq 0$. If $(x, y) \epsilon C_{\rho}$, then

$$
H\left[(x, y), I_{r}, y \geq 0\right] \geq 1-2 \rho / \pi r .
$$

Proof. It is easy to show that if $\phi$ is the angle at $(-r, 0)$ from $I_{r}$ to the circle $C$ through the points $(-r, 0),(r, 0)$ and $(x, y)$, then $H\left[(x, y), I_{r}, y \geq 0\right]=1-\phi / \pi$.

Now if $\rho / r=(1-\cos \phi) / \sin \phi$, then $C_{\rho}$ is contained in the region bounded by $C$ and the line $y=0$. Hence, by the minimum principle for harmonic functions we have for $(x, y) \epsilon C_{\rho}$

$$
H\left[(x, y), I_{r}, y \geq 0\right] \geq 1-(2 / \pi) \tan ^{-1}(\rho / r) \geq 1-2 \rho / \pi r,
$$

and the lemma is proved.

Lemma 5.2. Let $C_{r, \psi}$ denote the region $x^{2}+y^{2} \leq r^{2}, 0 \leq \tan ^{-1}(y / x) \leq \psi \leq 2 \pi$ and let $B_{r, \psi}$ denote the bounding radii of $C_{r, \psi}$. (If $\psi=2 \pi$, then $B_{r, \psi}$ denotes the line $0 \leq x \leq r, y=0$ ). If $\rho \leq r$ and if $(x, y) \epsilon C_{\rho, \psi}$, then

$$
H\left[(x, y), B_{r, \psi}, C_{r, \psi}\right] \geq 1-(4 / \pi)(\rho / r)^{\pi / \psi} .
$$

Proof. We first consider the case $\psi=\pi$. If $x^{2}+y^{2}=r^{2}$ and $y>0$, then as in lemma 5.1 we have $H\left[(x, y), I_{r}, y \geq 0\right]=1 / 2$. Hence, as we verify at once,

$$
H\left[(x, y), I_{r}, C_{\tau}\right]=2 H\left[(x, y), I_{r}, y \geq 0\right]-1 .
$$

If $(x, y) \epsilon C_{\rho, \pi}$, then by lemma 5.1 we have

$$
H\left[(x, y), I_{r}, C_{r}\right] \geq 1-(4 / \pi)(\rho / r) .
$$

The lemma can now be verified for the general case by mapping $C_{r, \pi}$ onto $C_{r, \psi}$ by means of the conformal transformation $(w / r)=(z / r)^{\psi / \pi}$. We omit the details.

Now by the maximum principle for harmonic functions, for given $\delta$ the maximum value of $\left|U\left(x_{1}, y_{1}\right)-U\left(x_{2}, y_{2}\right)\right|$ for $\left(x_{1}-x_{2}\right)^{2}+\left(y_{1}-y_{2}\right)^{2} \leq \delta^{2}$ occurs when either $\left(x_{1}, y_{1}\right)$ or $\left(x_{2}, y_{2}\right)$ belongs to $S$. If $\left(x_{1}, y_{1}\right) \epsilon S$, then for all $r$ such that $0<r \leq r_{0}$ there exists a circular sector $C_{r, \psi}$ containing at least one point of $R$ with vertex at $\left(x_{1}, y_{1}\right)$ with radius $r \geq \delta$ and angle $\psi$, such that $B_{r, \psi}$ the union of the bounding radii is disjoint from $R$. Since the theorem is trivially true if $\left(x_{2}, y_{2}\right) \epsilon S$, we assume $\left(x_{2}, y_{2}\right) \epsilon R$. Let $\Omega_{1}$ denote the closure of the connected component of $\left(x_{2}, y_{2}\right)$ for the region $\Omega \cap C_{r, \psi}$. Evidently $\Omega_{1} c C_{r, \psi}$. Since for $(x, y) \epsilon \Omega_{1} \cap S$, we have

$$
H\left[(x, y), B_{r, \psi}, C_{r, \psi}\right] \leq 1=H\left[(x, y), \Omega_{1} \cap S, \Omega_{1}\right]
$$

and for $(x, y)$ contained in $\Omega_{1}$ and on the arc of $C_{r, \psi}$, we have

$$
H\left[(x, y), B_{r, \psi}, C_{r_{\bullet} \psi}\right]=0,
$$


then for all $(x, y) \epsilon \Omega_{1}$ it follows that

$$
H\left[(x, y), B_{r, \psi}, C_{r, \psi}\right] \leq H\left[(x, y), \Omega_{1} \cap S, \Omega_{1}\right] .
$$

But by lemma 5.2 we have

$$
H\left[\left(x_{2}, y_{2}\right), B_{r, \psi}, C_{r, \psi}\right] \geq 1-(4 / \pi)(\delta / r)^{\pi / \psi}
$$

since the lemma is obviously true if we rotate the sector.

Therefore, we have

$$
\left|u\left(x_{2}, y_{2}\right)-u\left(x_{1}, y_{1}\right)\right| \leq \omega(r) H\left[\left(x_{2}, y_{2}\right), C_{r, \psi} \cap S, \Omega\right]+M H\left[\left(x_{2}, y_{2}\right), S-C_{r, \psi}, \Omega\right] \leq \omega(r)+(4 M / \pi)(\delta / r)^{\pi / \psi} .
$$

Now by the assumptions on $\delta$ we have $D(\delta / D)^{\pi /(\pi+\psi)} \leq D\left(r_{0} / D\right)=r_{0}$. Hence, we can choose $r=D(\delta / D)^{\pi /(\pi+\psi)} \leq r_{0}$, and the theorem follows.

We now consider the case where $\Omega$ is an arbitrary simply connected region. We first prove the following general theorem, which is essentially a formulation for harmonic functions which is equivalent to Carathéodory's form of the theorem of Milloux for analytic functions:

Theorem 5.2. Let $G$ be a Jordan subregion of $|z|<1$, whose boundary consists of a Jordan arc $\alpha_{1}$ which passes through $z=0$ plus an arc $\alpha_{2}$ of $\Gamma:|z|=1$. Let $u(z)$ be harmonic and bounded in $G$, continuous in the corresponding closed region except at the end points of $\alpha_{1}$ and $\alpha_{2}$, equal to unity in the interior points of $\alpha_{1}$ and to zero in the interior points of $\alpha_{2}$. Then in every point $z$ of $G$ we have

$$
u(z) \geq 1-(4 / \pi) \tan ^{-1}|z|^{1 / 2} .
$$

Proof. Let $v(z)$ be conjugate to $u(z)$ in $G$, and let $f(z) \equiv \exp [-u(z)-i v(z)]$. Except perhaps at the end points of the arcs, on $\alpha_{1}$ we have $|f(z)|=e^{-1}$, and on $\alpha_{2}$ we have $|f(z)|=1$; on these open arcs $|f(z)|$ is continuous in the two-dimensional sense. Then by the form of Milloux's theorem presented by Carathéodory, [1], 354, we have for $z \in G$

as we were to prove.

$$
\log |f(z)|=-u(z) \leq-1+(4 / \pi) \tan ^{-1}|z|^{1 / 2}
$$

Theorem 5.3. Let $\Omega$ denote a bounded simply connected closed region with interior $R$ and boundary S. Let $u(x, y)$ be harmonic in $R$, continuous in $\Omega$ and having modulus of continuity $\omega(\delta)$ on $S$. If $D$ is any positive constant, then the modulus of continuity of $u(x, y)$ in $\Omega$ satisfies the inequality

for all $\delta \leq D$.

$$
\omega^{*}(\delta) \leq \omega\left[D(\delta / D)^{1 / 3}\right]+(4 M / \pi)(\delta / D)^{1 / 3}
$$

Proof. As in the proof of theorem 5.1, it is sufficient to show that if $C_{r}$ is a circle with center $\left(x_{1}, y_{1}\right)$ on $S$ and radius $r$ and if $\left(x_{2}, y_{2}\right) \epsilon \Omega$ and $\left(x_{1}-x_{2}\right)^{2}+\left(y_{1}-y_{2}\right)^{2} \leq \delta^{2} \leq r^{2}$, then

$$
H\left[\left(x_{2}, y_{2}\right), C_{r} \cap S, \Omega\right] \geq 1-(4 / \pi)(\delta / r)^{1 / 2} .
$$

The theorem then follows if we let $r=D(\delta / D)^{1 / 3}$ since $\delta \leq D$ and hence $D(\delta / D)^{1 / 3} \geq \delta$.

Now, if $\Omega$ is a Jordan region, (5.4) follows at once from theorem 5.2, since $\tan ^{-1}|z|^{1 / 2} \leq|z|^{1 / 2}$ for $|z| \leq 1$. We indicate the modifications necessary to include the case where $\Omega$ is an arbitrary simply connected region.

Let $O$ be a boundary point of $\Omega$, let $C_{\tau}$ be the circle $|z|=r$, and let $z_{0}$ be a point interior to $\Omega$ and to $C_{r}$. If no point of $\Omega$ lies exterior to $C_{r}$, then a function $v(z)$ harmonic in $R$ and continuous in $\Omega$ equal to unity on $S$ is identically unity in $\Omega$ so we have $v\left(z_{0}\right)=1$. We proceed to study the contrary case. If points of $R$ lie exterior to $C_{r}$, such points can be joined to $z_{0}$ by a Jordan arc lying wholly in $R$, so at least one arc of $C_{r}$ lies in $R$. Let the totality of mutually disjoint ares of $C_{r}$ in $R$ be $A_{1}, A_{2}$, . . . Denote by $R_{0}$ the subregion of $R$ interior to $C_{r}$ containing $z_{0}$ and by $R_{1}$ the sum of $R_{0}$, its reflection (inverse) in $C_{r}$, and the arcs $A_{k}$ which form part of the boundary of $R_{0}$; if an arc of $C_{r}$ is part of the boundary of $R$, it does not belong to $R_{1}$. We modify $R_{1}$ by 
adjoining to $R_{1}$ the interior of each Jordan curve that can be drawn in $R_{1}$ and to which $O$ and $z_{0}$ are exterior. Denote this new region by $R_{2}$. Then $R_{2}$ contains $z_{0}$, is simply connected, and has $O$ as either an exterior or a boundary point. The subregion $R_{3}$ of $R_{2}$ interior to $C_{r}$ is also simply connected, and part of its boundary is an open arc $A_{0}$ of $C_{r}$ of which every point is accessible; we choose $A_{0}$ as the largest such arc, so that every boundary point of $R_{3}$ not on $A_{0}$ is either a boundary point of $R$ interior to $C_{r}$ or is a point of $C_{r}$ not contained in an arc of $C_{r}$ consisting wholly of accessible boundary points of $R_{3}$. By a conformal map of $R_{3}$ onto the interior of a circle $\gamma$ (in which $A_{0}$ necessarily corresponds to an arc of $\gamma$ ) it is clear that $H\left(z, A_{0}, R_{3}\right)$ exists and is unique; this function takes the boundary value unity at every point of $A_{0}$ and the boundary value zero at every boundary point of $R_{3}$ not on the closure of $A_{0}$. Carathéodory's proof of Milloux's theorem is valid for the region $R_{3}$, the distance from $z_{0}$ to the boundary of $R_{3}$ is not greater than $\left|z_{0}\right|$, so (5.4) is valid for an arbitrary simply connected region, and the theorem follows.

The modulus of continuity of a function $F(x, y)$ on any subset $\Omega_{L}$ of $L[h, k]$ is defined by

$$
\omega_{L}(\delta)=L U B\left|F\left(x_{1}, y_{1}\right)-F\left(x_{2}, y_{2}\right)\right|,
$$

where $\left(x_{1}, y_{1}\right),\left(x_{2}, y_{2}\right) \epsilon \Omega_{L}$, and where $\left(x_{1}-x_{2}\right)^{2}+\left(y_{1}-y_{2}\right)^{2} \leq \delta^{2}$.

For d.h. functions in a rectangle we prove the following theorem:

Theorem 5.4. Let $\Omega$ denote the rectangle $0 \leq x \leq a, 0 \leq y \leq b$, where $(a, b) \epsilon L[h, k]$. Let $\Omega_{L}$ denote the subset of $L[h, k]$ contained in $\Omega$. If $U(x, y)$ is d.h. in $R_{L}$, the interior of $\Omega_{L}$, and has modulus of continuity $\omega(\delta)$ on $S_{L}$, the boundary of $\Omega_{L}$, then for $\delta \leq r=(a b)^{1 / 2}$ the modulus of continuity of $U(x, y)$ in $\Omega_{L}$ satisfies the inequality

$$
\omega_{L}^{*}(\delta) \leq \omega\left[2^{1 / 2}\left\{(r \delta)^{1 / 2}+h+k\right\}\right]+(M / 2) \nu(1 / 2)(\delta / r)^{1 / 2}
$$

where $M$ and $\nu(1 / 2)$ are defined by (5.2) and (4.6), respectively.

Proof. As in the proof of theorem 5.1 the maximum value of $\left|U\left(x_{1}, y_{1}\right)-U\left(x_{2}, y_{2}\right)\right|$ for $\left(x_{1}-x_{2}\right)^{2}+\left(y_{1}-y_{2}\right)^{2} \leq \delta^{2}$ is assumed when either $\left(x_{1}, y_{1}\right)$ or $\left(x_{2}, y_{2}\right)$ belongs to $S_{L}$. Let us assume that $\left(x_{1}, y_{1}\right) \epsilon S_{L}$. Now if $\left(x_{2}, y_{2}\right)$ also belongs to $S_{L}$ the theorem is trivial, since $\delta \leq r$. We therefore assume $\left(x_{2}, y_{2}\right) \epsilon R_{L}$.

Let $p$ denote a straight line containing $\left(x_{1}, y_{1}\right)$ and including one of the sides of the rectangle. Let $C$ denote the closed interior of a semicircle with center at $\left(x_{1}, y_{1}\right)$, with radius $\delta_{1}=2^{1 / 2}\left\{(\delta r)^{1 / 2}+\right.$ $h+k\}$, with bounding diameter included in $p$ and containing at least one point of $R_{L}$. Since $\delta \leq r$ we have $\delta_{i} \geq 2^{1 / 2} \delta$. Evidently there exists a rectangle $T$ included in $C$ with one side contained in $p$ and with vertices contained in $L[h, k]$ such that the sides perpendicular to $p$ are at least half as long as those parallel to $p$ and such that the latter sides have length at least $2(\delta r)^{1 / 2}$.

By (4.2) and (4.3) we have

$$
H_{L}\left[(x, y), C \cap S_{L}, \Omega_{L}\right] \geq H_{L}\left[(x, y), T \cap S_{L}, \Omega_{L}\right] \geq H_{L}\left[(x, y), T \cap S_{L}, \Omega_{L} \cap T\right] .
$$

Now, for $(x, y) \epsilon S_{L} \cap T$ we have

$$
H_{L}[(x, y), T \cap p, T] \leq 1=H_{L}\left[(x, y), T \cap S_{L}, \Omega_{L} \cap T\right] .
$$

Moreover, since the intersection of $p$ and the boundary of $T \cap \Omega_{L}$ is contained in $T \cap S_{L}$ we have $H_{L}[(x, y), T \cap p, T]=0$ for all points of the boundary of $\Omega_{L} \cap T$ not contained in $S_{L} \cap T$. Therefore, for all points of the boundary of $T \cap \Omega_{L}$ we have

$$
H_{L}\left[(x, y), T \cap S_{L}, \Omega_{L} \cap T\right] \geq H_{L}[(x, y), T \cap p, T] .
$$

It follows that for all $(x, y) \epsilon T \cap \Omega_{L}$ we have

$$
H_{L}\left[(x, y), T \cap S_{L}, \Omega_{L} \cap T\right] \geq H_{L}[(x, y), T \cap p, T] .
$$


Now since $\left(x_{2}, y_{2}\right) \epsilon T \cap \Omega_{L}$, we have by theorem 4.1

Hence,

$$
H_{L}\left[\left(x_{2}, y_{2}\right), T \cap p, T\right] \geq 1-(1 / 2) \nu(1 / 2)(\delta / r)^{1 / 2} .
$$

Therefore,

$$
H_{L}\left[\left(x_{2}, y_{2}\right), S_{L}-\left(S_{L} \cap C\right), \Omega_{L}\right] \leq(1 / 2) \nu(1 / 2)(\delta / r)^{1 / 2} .
$$

$$
\left|U\left(x_{1}, y_{1}\right)-U\left(x_{2}, y_{2}\right)\right| \leq \omega\left[2^{1 / 2}\left\{(r \delta)^{1 / 2}+h+k\right\}\right]+(M / 2) \nu(1 / 2)(\delta / r)^{1 / 2},
$$

and the theorem follows.

In the above proof if $h=k$, one could have obtained a rectangle with the desired properties by letting $C$ have radius $2^{1 / 2}\left[(r \delta)^{1 / 2}+h\right]$. Therefore, we have

Corollary: If $h=k$, then

$$
\omega_{L}^{*}(\delta) \leq \omega\left[2^{1 / 2}\left\{(r \delta)^{1 / 2}+h\right\}\right]+(M / 2) \nu(1 / 2)(\delta / r)^{1 / 2} .
$$

\section{Error Estimate for the Rectangle In Terms of the Modulus of Continuity on the Boundary}

In this section we obtain an error bound for the rectangle of section 3 under the assumption that the function $f(x, y)$, which determines the boundary values, has modulus of continuity $\omega(\delta)$ on $S$. The function $f_{3}(x, y)$ defined in section 3 is also continuous, and we denote by $\omega_{3}(\delta)$ its modulus of continuity for $0 \leq x \leq a, y=0$.

Let $U_{3}(x, y)$ and $u_{3}(x, y)$ be given by (3.3) and (3.5), respectively. We define the function $f_{5}(x)$ by the partial sum

$$
f_{5}(x)=\sum_{n=1}^{A} d_{A, n} A_{n} \sin (n \pi x / a),
$$

where the $A_{n}$ are the Fourier coefficients for $f_{3}(x, 0)=g(x)$ as defined by (3.4) and where the coefficients $d_{A, n}$ are summation coefficients defined by Jackson [7, p. 9]. Let $u_{5}(x, y)$ and $U_{5}(x, y)$ denote respectively the solutions of the Dirichlet problem and its difference analog vanishing on $S$ except for $y=0$. If $y=0$, the values are determined by $f_{5}(x)$. We now prove

Theorem 6.1. If $(x, y) \epsilon \Omega_{L}$ and $0 \leq x \leq a, \epsilon \leq y \leq b$, then

$$
\left|U_{5}(x, y)-u_{5}(x, y)\right| \leq 8 M^{*} C \lambda(h / a)^{2}(\epsilon / a)^{-3},
$$

where

$$
\lambda=\frac{6 e}{\alpha^{3} \pi^{3}}+\frac{27 \epsilon / a}{\alpha^{2} \pi^{2} e^{2}}, \quad M^{*}=\underset{0 \leq x \leq a}{\operatorname{Max}}|g(x)|
$$

and where $C$ and $\alpha$ are determined by (3.10) and (3.12), respectively.

Proof. It can be verified that

$$
\begin{gathered}
u_{5}(x, y)=\sum_{n=1}^{A} d_{A, n} A_{n} \sin (n \pi x / a) \Gamma_{n}(y), \\
U_{5}(x, y)=\sum_{n=1}^{A} d_{A, n} A_{n} \sin (n \pi x / a) \Gamma_{m}(y),
\end{gathered}
$$

where $m$ and $n$ are related by (3.7), and where $\Gamma_{m}(y)$ is defined by (3.16). Evidently we have

$$
\left|U_{5}(x, y)-u_{5}(x, y)\right| \leq \sum_{n=1}^{A}\left|d_{A, n} A_{n}\right| \cdot\left|\Gamma_{m}(y)-\Gamma_{n}(y)\right| .
$$

By lemma 3.3 we have

$$
\left|\frac{d}{d m} \Gamma_{m}(y)\right| \leq(\pi y / a) \operatorname{coth}(m \pi b / a) \exp (-m \pi y / a), \quad(0 \leq y \leq b), \quad(m \geq 0) .
$$


By lemma 3.2 we have $m \geq \alpha$ for $1 \leq n \leq A$, and

$$
\left|\frac{d}{d m} \Gamma_{m}(y)\right| \leq(\pi y / a) \operatorname{coth}(\alpha \pi b / a) \exp (-\alpha n \pi y / a) .
$$

Using lemma 3.1 we get, as in the proof of lemma 3.5,

Therefore, we have

$$
\left|\Gamma_{m}(y)-\Gamma_{n}(y)\right| \leq C \alpha e(h / a)^{2}(\pi y / a) n^{3} \exp (-\alpha n \pi y / a) .
$$

$$
\sum_{n=1}^{A}\left|\Gamma_{m}(y)-\Gamma_{n}(y)\right| \leq \sum_{n=1}^{\infty}\left|\Gamma_{m}(y)-\Gamma_{n}(y)\right| \leq C \alpha e(h / a)^{2}(\pi y / a) \sum_{n=1}^{\infty} n^{3} \exp (-\alpha n \pi y / a),
$$

which is convergent for $y>0$.

Now the following statement can be verified easily: if $G(x)$ is a continuous nonnegative function for $x \geq 0$, nondecreasing for $0 \leq x \leq X$, and nonincreasing for $X \leq x$, then

$$
\sum_{n=1}^{\infty} G(n) \leq \int_{0}^{\infty} G(t) d t+G(X)
$$

Therefore, since for $\nu>0$ we have

it follows that

$$
\operatorname{Max}_{x \geq 0}\left[x^{3} \exp (-\nu x)\right]=(3 / \nu e)^{3}
$$

$$
\sum_{n=1}^{\infty} n^{3} \exp (-\alpha n \pi y / a) \leq \int_{0}^{\infty} t^{3} \exp (-\alpha \pi y t / a) d t+(3 a / \alpha \pi y e)^{3} \leq 6(a / \alpha \pi y)^{4}+(3 a / \alpha \pi y e)^{3} .
$$

Therefore, we get

$$
\sum_{n=1}^{A}\left|\Gamma_{m}(y)-\Gamma_{n}(y)\right| \leq \lambda C(h / a)^{2}(y / a)^{-3} \leq \lambda C(h / a)^{2}(\epsilon / a)^{-3}
$$

since $y \geq \epsilon$.

For the Fourier coefficients, since $|g(t)| \leq M^{*}$, we have

$$
\left|A_{n}\right|=\left|(2 / a) \int_{0}^{a} g(t) \sin (n \pi t / a) d t\right| \leq(2 / a) \int_{0}^{a}|g(t)| d t \leq 2 M^{*} .
$$

Jackson [6] proved that $\left|1-d_{\boldsymbol{A}, n}\right| \leq 3 n / A \leq 3$. Hence

$$
\left|d_{A, n}\right| \leq 4
$$

From (6.6), (6.9), (6.10), and (6.11) the theorem now follows.

Corollary. For $(x, y) \epsilon \Omega_{L}$ and $0 \leq x \leq a, \epsilon \leq y \leq b$ we have

$$
\left|U_{3}(x, y)-u_{3}(x, y)\right| \leq 8 M^{*} C \lambda(h / a)^{2}(\epsilon / a)^{-3}+2 K \omega_{3}(2 h),
$$

where $K$ is a positive absolute constant less than ${ }^{10} 3$.

Proof.

$$
\left|U_{3}(x, y)-u_{3}(x, y)\right| \leq\left|U_{3}(x, y)-U_{5}(x, y)\right|+\left|U_{5}(x, y)-u_{5}(x, y)\right|+\left|u_{5}(x, y)-u_{3}(x, y)\right| .
$$

By the maximum and minimum principles for harmonic and d.h. functions, we have for $(x, y) \epsilon \Omega_{L}$

$$
\left|U_{3}(x, y)-U_{5}(x, y)\right|,\left|u_{3}(x, y)-u_{5}(x, y)\right| \leq \operatorname{Max}_{0 \leq x \leq a}\left|f_{5}(x)-g(x)\right| .
$$

Using a theorem of Jackson [7, p. 7], we obtain

$$
\operatorname{Max}_{0 \leq x \leq a}\left|f_{5}(x)-g(x)\right| \leq K \omega_{3}(2 a / A),
$$

10 See Jackson $[7$, p. 7]. 
where $K$ is a positive absolute constant less than 3. The corollary now follows from the theorem.

We shall now derive an error bound for the original problem where we no longer assume that the boundary values vanish at the corners or along the sides of the given rectangle.

Theorem 6.2. For all $(x, y) \epsilon \Omega_{L}$, we have

where

$$
|U(x, y)-u(x, y)| \leq \operatorname{Max}\left\{I ; 2 \omega_{L}^{*}\left[r\left(\frac{h k}{r^{2}}\right)^{2 / 7}\right]\right\},
$$

$$
\begin{aligned}
I= & 16 M\left[\lambda_{a} C_{a} \sigma^{-6 / 7}(a / b)^{9 / 14}(h / a)^{2 / 7}+\lambda_{b} C_{b} \sigma^{6 / 7}(b / a)^{9 / 14}(k / b)^{2 / 7}\right]-+ \\
& 4 K\{\omega(2 h)+\omega(2 k)+2 M[h / a+k / b]\}, \\
r= & (a b)^{1 / 2} \\
C_{a}= & \frac{\pi^{2}\left(1+\sigma^{2}\right)}{24 \alpha e} \operatorname{coth}(\alpha \pi b / a), \quad C_{b}=\frac{\pi^{2}\left(1+\sigma^{-2}\right)}{24 \beta e} \operatorname{coth}(\beta \pi a / b) \\
\alpha= & (2 / \pi \sigma) \sinh ^{-1}(\sigma), \quad \beta=(2 \sigma / \pi) \sinh ^{-1}\left(\sigma^{-1}\right) \\
\lambda_{a}= & \left.\frac{6 e}{\alpha^{3} \pi^{3}}+\frac{27 / 2}{\alpha^{2} \pi^{2} e^{2}}\right\} \\
\lambda_{b}= & \left.\frac{6 e}{\beta^{3} \pi^{3}}+\frac{27 / 2}{\beta^{2} \pi^{2} e^{2}}\right\} \\
M= & \operatorname{Max}_{(x, y) \epsilon S} u(x, y)-\operatorname{Min}_{(x, y) \epsilon S} u(x, y) \\
\omega_{L}^{*}(\delta)= & \omega\left[2^{1 / 2}\left\{(r \delta)^{1 / 2}+h+k\right\}\right]+(M / 2) \nu(1 / 2)(\delta / r)^{1 / 2}
\end{aligned}
$$

and $\omega(\delta)$ is the modulus of continuity of $u(x, y)$ on $S$. The function $\nu(b / a)$ is defined by (4.6) and $\nu(1 / 2)$ equals 3.97 , approximately.

\section{Proof.}

Lemma 6.1. If $u_{1}(x, y)$ is defined by (3.1), then for all $(x, y) \in \Omega$ we have $\left|u(x, y)-u_{1}(x, y)\right| \leq M$.

Proof. Since $u(x, y)$ and $u_{1}(x, y)$ are harmonic, the maximum of $\left|u(x, y)-u_{1}(x, y)\right|$ is assumed on $S$. We can assume, without loss of generality that the maximum occurs at a point whose ordinate is zero. By (3.1) we have

$$
u_{1}(x, 0)=(1-x / a) u(0,0)+(x / a) u(a, 0),
$$

and

$$
\left|u(x, 0)-u_{1}(x, 0)\right| \leq|1-x / a| \cdot|u(x, 0)-u(0,0)|+|x / a| \cdot|u(x, 0)-u(a, 0)| .
$$

But,

$$
|u(x, 0)-u(0,0)|,|u(x, 0)-u(a, 0)| \leq \operatorname{Max}_{0 \leq x \leq a} u(x, 0)-\operatorname{Min}_{0 \leq x \leq a} u(x, 0) \leq M,
$$

and the lemma follows.

The above result cannot be improved as one can show by considering the case where $u_{1}(x, y)$ is identically zero and $u(x, y) \geq 0$.

From lemma 6.1 we conclude that $\left|u_{3}(x, y)\right| \leq M$. In particular we have $|g(x)| \leq M$; hence,

$$
M^{*} \leq M \text {. }
$$

Lemma 6.2.

$$
\omega_{3}(\delta) \leq \omega(\delta)+\delta M / a
$$

Proof. Evidently we have $\omega_{3}(\delta) \leq \omega(\delta)+\omega_{1}(\delta)$, where $\omega_{1}(\delta)$ denotes the modulus of con- 
tinuity of $u_{1}(x, 0)$ considered as a function of $x$. But since

$$
u_{1}(x+\delta, 0)-u_{1}(x, 0)=(\delta / a)[u(a, 0)-u(0,0)],
$$

we have $\omega_{1}(\delta) \leq \delta M / a$, and the lemma follows.

Lemм. 6.3. If $\epsilon \leq x \leq a-\epsilon, \epsilon \leq y \leq b-\epsilon,(x, y) \epsilon \Omega_{L}$, then

$|U(x, y)-u(x, y)| \leq 16 M\left\{\lambda_{a} C_{a}(h / a)^{2}(\epsilon / a)^{-3}+\lambda_{b} C_{b}(k / b)^{2}(\epsilon / b)^{-3}\right\}+4 K[\omega(2 h)+\omega(2 k)+2 M(h / a+k / b)]$.

Proof. We observe that $|U(x, y)-u(x, y)|=\left|U_{2}(x, y)-u_{2}(x, y)\right|$. The function $\left[U_{2}(x, y)\right.$ $\left.-u_{2}(x, y)\right]$ is the sum of $\left[U_{3}(x, y)-u_{3}(x, y)\right]$ and three other terms of similar type. The lemma follows from (6.12), from (6.20) and from lemma 6.2. (We note that from the hypothesis we have $\epsilon / a, \epsilon / b \leq 1 / 2$.)

Lemma 6.3 affords us an error bound for those points of $\Omega_{L}$ which are at a distance of not less than $\epsilon$ from the boundary. In order to obtain a uniform error bound we now consider those points which are within $\epsilon$ of the boundary.

Since $4 / \pi<\nu(1 / 2) / 2$ it follows from theorem 5.1, (with $D=r$ ), and theorem 5.4 that the moduli of continuity of $U(x, y)$ and $u(x, y)$ do not exceed the upper bound for $\omega_{L}^{*}(\delta)$ given by theorem 5.4, provided $\delta \leq(a b)^{1 / 2}$. If $(x, y) \epsilon \Omega_{L}$ and if $(x, y)$ is within $\epsilon$ of a point of $S$, then there exists a point of $S_{L}$ at a distance not greater than $\epsilon$ from $(x, y)$. We remark that every point of $\Omega_{L}$ is within $(a b)^{1 / 2}$ of some point of $S_{L}$. Since $U(x, y)=u(x, y)$ on $S_{L}$ we have

$$
|U(x, y)-u(x, y)| \leq 2 \omega_{L}^{*}(\epsilon) .
$$

If $I$ denotes the right member of $(6.22)$, then we have

$$
|U(x, y)-u(x, y)| \leq \operatorname{Max}\left\{I ; 2 \omega_{L}^{*}(\epsilon)\right\} .
$$

In order that the error in $\Omega_{L}$ should approach zero with the highest power of $\gamma=(h k)^{1 / 2}$ whenever $\omega(\delta) / \delta$ is bounded as a function of $\delta$, we choose $\epsilon=r\left(h k / r^{2}\right)^{2 / 7}$. Evidently we have

$$
\epsilon / a=\sigma^{2 / 7}(h / a)^{4 / 7}(b / a)^{3 / 14}, \quad \epsilon / b=\sigma^{-2 / 7}(k / b)^{4 / 7}(a / b)^{3 / 14} .
$$

The theorem now follows from (6.23), lemma 6.3, and theorem 5.4.

For the special case of the unit square theorem 6.2 and theorem 5.4, corollary, give

Corollary. If $a=b=\sigma=1$, then we have

where

$$
|U(x, y)-u(x, y)| \leq \operatorname{Max}\left\{J_{1} ; J_{2}\right\},
$$

$$
\begin{array}{ll}
J_{1}=32 M \lambda C h^{2 / 7}+8 K[\omega(2 h)+2 M h], & C=\left(\pi^{2} / 12 \alpha e\right) \operatorname{coth}(\alpha \pi) \sim .572, \\
J_{2}=2 \omega\left[2^{1 / 2}\left(h^{2 / 7}+h\right)\right]+M \nu(1 / 2) h^{2 / 7}, & \alpha=(2 / \pi) \sinh ^{-1}(1) \sim .561, \\
\lambda & =\frac{6 e}{\alpha^{3} \pi^{3}}+\frac{27 / 2}{\alpha^{2} \pi^{2} e^{2}} \sim 3.566 .
\end{array}
$$

Substituting numerical values, we obtain

$$
J_{1} \leq 66 M h^{2 / 7}+24 \omega(2 h)+48 M h, \quad J_{2} \leq 2 \omega\left[2^{1 / 2}\left(h^{2 / 7}+h\right)\right]+4 M h^{2 / 7} .
$$

The above expressions for $J_{1}$ and $J_{2}$ represent a slight improvement in the formulas previously given by the authors [14].

\section{Other Regions}

In this section we consider regions of the type described in section 2 other than rectangles. The case of two overlapping rectangles is studied in detail as an illustration of the method 
which can be extended to more complicated regions. We first prove a theorem valid for two overlapping regions whether rectangles or not.

Theorem 7.1. Let $\Omega=\Omega^{\prime} \cup \Omega^{\prime \prime}$, where $R^{\prime} \cap R^{\prime \prime}$ is not empty, and where neither region includes the other. Let $u(x, y)$ and $U(x, y)$ denote, respectively, the solution of the Dirichlet problem and its finite difference analog for $\Omega$. Let $\Omega_{L}, \Omega_{L}^{\prime}$, and $\Omega_{L}^{\prime \prime}$ denote the points of $L[h, k]$ belonging to $\Omega, \Omega^{\prime}$, and $\Omega^{\prime \prime}$, respectively. Let $T_{L}^{\prime}=S_{L}^{\prime} \cap R_{L}^{\prime \prime}, T_{L}^{\prime \prime}=S_{L}^{\prime \prime} \cap R_{L}^{\prime}$, and let

$$
\mu_{1}=\underset{(x, y) \in T_{L}^{\prime}}{\operatorname{Max}_{L}} H_{L}\left[(x, y), T_{L}^{\prime \prime}, \Omega_{L}^{\prime \prime}\right], \quad \mu_{2}=\underset{(x, y) \in T_{L}^{\prime \prime}}{\operatorname{Max}_{L}} H_{L}\left[(x, y), T_{L}^{\prime}, \Omega_{L}^{\prime}\right] .
$$

Next, let $U^{\prime}$ and $U^{\prime \prime}$ denote functions d.h. in $\Omega_{L}^{\prime}$ and $\Omega_{L}^{\prime \prime}$, respectively, and equal to $u$ on $S_{L}^{\prime}$ and $S_{L}^{\prime \prime}$, respectively. Then for $(x, y) \epsilon \Omega_{L}$, we have

$$
|U(x, y)-u(x, y)| \leq \operatorname{Max}(A, B),
$$

where

$$
\begin{array}{ll}
A=\frac{E^{\prime}\left(1+\mu_{1}-\mu_{1} \mu_{2}\right)+E^{\prime \prime}}{1-\mu_{1} \mu_{2}}, & E^{\prime}=\operatorname{Max}_{(x, y) \in R_{L}^{\prime}}\left|U^{\prime}(x, y)-u(x, y)\right|, \\
B=\frac{E^{\prime \prime}\left(1+\mu_{2}-\mu_{1} \mu_{2}\right)+E^{\prime}}{1-\mu_{1} \mu_{2}}, & E^{\prime \prime}=\operatorname{Max}_{(x, y) \in R_{L}^{\prime \prime}}\left|U^{\prime \prime}(x, y)-u(x, y)\right| .
\end{array}
$$

Proof. Let

$$
F^{\prime}=\underset{(x, y) \epsilon T_{L}^{\prime}}{\operatorname{Max}}|U(x, y)-u(x, y)|, \quad F^{\prime \prime}=\underset{(x, y) \in T_{L}^{\prime \prime}}{\operatorname{Max}}|U(x, y)-u(x, y)| .
$$

Clearly, for $(x, y) \epsilon T_{L}^{\prime}$

$$
\begin{aligned}
|U(x, y)-u(x, y)| \leq\left|U(x, y)-U^{\prime \prime}(x, y)\right|+\mid U^{\prime \prime}(x, y) & -u(x, y) \mid \\
& \leq \mu_{1} \operatorname{Max}_{(x, y) \in T_{L}^{\prime \prime}}\left|U(x, y)-U^{\prime \prime}(x, y)\right|+\left|U^{\prime \prime}(x, y)-u(x, y)\right| .
\end{aligned}
$$

Hence, by the maximum and minimum principles for d.h. functions $F^{\prime} \leq \mu_{1} F^{\prime \prime}+E^{\prime \prime}$. Similarly, $F^{\prime \prime} \leq \mu_{2} F^{\prime}+E^{\prime}$. Therefore,

$$
F^{\prime} \leq \frac{\mu_{1} E^{\prime}+E^{\prime \prime}}{1-\mu_{1} \mu_{2}}, \quad F^{\prime \prime} \leq \frac{\mu_{2} E^{\prime \prime}+E^{\prime}}{1-\mu_{1} \mu_{2}} .
$$

By the maximum and minimum principles,

$$
\operatorname{Max}_{(x, y) \in \Omega_{L}^{\prime}}|U(x, y)-u(x, y)| \leq E^{\prime}+F^{\prime}, \quad \operatorname{Max}_{(x, y) \in \Omega_{L}^{\prime \prime}}|U(x, y)-u(x, y)| \leq E^{\prime \prime}+F^{\prime \prime},
$$

and the theorem follows.

The proof of this theorem was, of course, motivated by consideration of the difference analog of the Schwarz Alternating Process. Indeed, in order to solve the difference equation for $\Omega_{L}$, one might guess values for $U(x, y)$ on $S_{L}{ }^{\prime} \cap R_{L}{ }^{\prime \prime}$ and then solve the difference equation in $R_{L}{ }^{\prime}$ obtaining in particular values on $S_{L}{ }^{\prime \prime} \cap R_{L}{ }^{\prime}$. Next one solves the difference equation for $R_{L}^{\prime \prime}$, using the computed values on $S_{L}^{\prime \prime} \cap R_{L}{ }^{\prime}$, and obtains new values for $S_{L}^{\prime} \cap R_{L}{ }^{\prime \prime}$. This process can be repeated and the successive values thus obtained converge to the exact solution of the difference equation. Moreover, the rapidity of convergence can be estimated if $\mu_{1}$ and $\mu_{2}$ are known. In fact, one can show that after a complete iteration the maximum error is reduced by a factor of $\left(1-\mu_{1} \mu_{2}\right)$.

If for $\Omega^{\prime}$ and $\Omega^{\prime \prime}$ the quantities $E^{\prime}$ and $E^{\prime \prime}$ are known whenever the modulus of continuity and oscillation of $u(x, y)$ are known on $S^{\prime}$ and $S^{\prime \prime}$, then by using theorem 5.1 or theorem 5.3, $E^{\prime}$ and $E^{\prime \prime}$ can be computed, provided the modulus of continuity of $u(x, y)$ on $S$ is known. If, moreover, $\mu_{1}$ and/or $\mu_{2}$ are known, an error bound for the composite region can be obtained.

We consider now the case of two overlapping rectangles $\Omega_{1}$ and $\Omega_{2}$ with sides parallel to the coordinate axes. If the intersection of the interiors is not empty, and if neither region is a 
subset of the other, then the cases $\mathrm{A}$ through $\mathrm{F}$ shown in figure 1 occur.
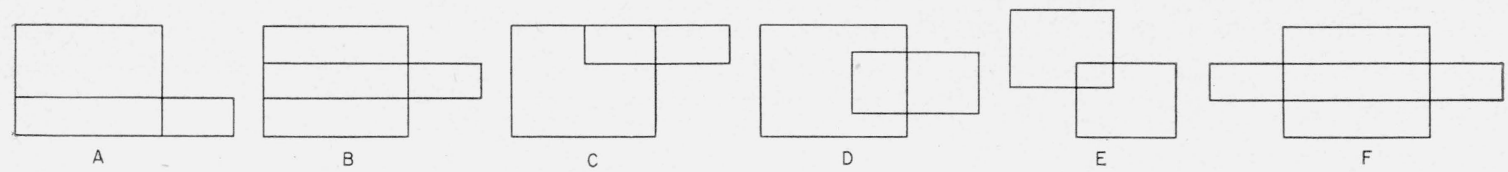

Figure 1.

The composite regions of cases $\mathrm{C}$ and $\mathrm{D}$ are of the same type as those of cases $\mathrm{A}$ and $\mathrm{B}$, respectively. The problem of determining $\mu_{1}$ and $\mu_{2}$ become problems in d.h.m. for the rectangle of the types shown in figure 2 .

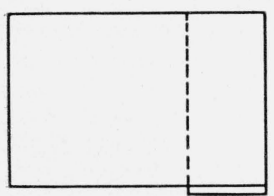

A

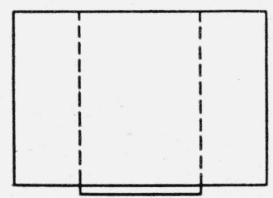

$B_{1}$

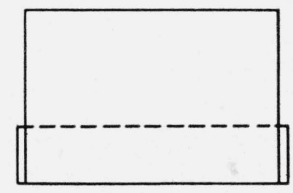

$\mathrm{B}_{2}$

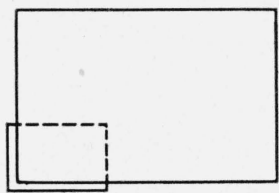

$E$

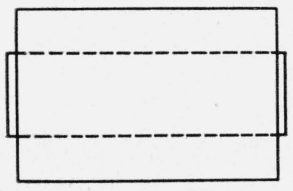

$\mathrm{F}$

Figure 2.

Upper bounds for d.h.m. are required in points on the closed dotted lines for the open arcs on the boundary indicated by heavy lines.

By (4.3), these upper bounds are not greater than the upper bounds for the problems shown in figure 3 .
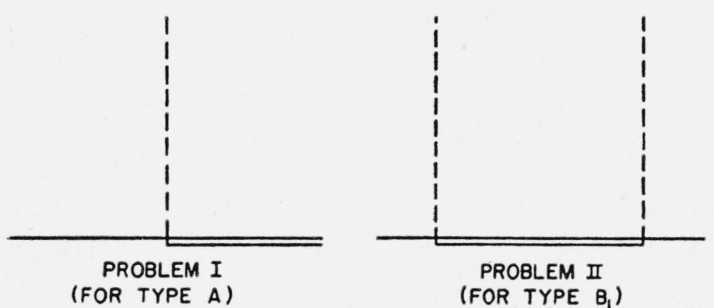
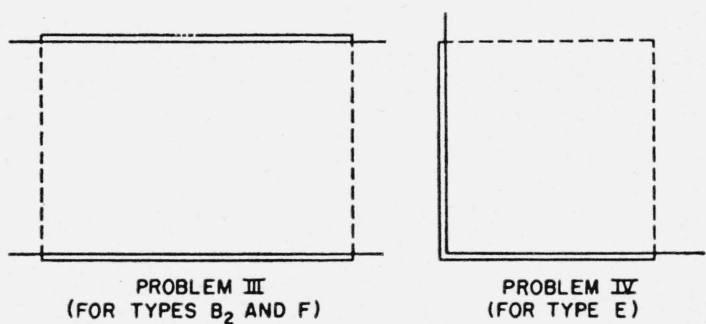

Figure 3.

For problem I, since the harmonic measure of the open arc is required, $\frac{1}{2}$ is an upper bound on the dotted line, by symmetry.

Theorem 7.2. Let $\Omega_{L}$ be bounded in part by a segment of a line $l$. Let $R_{L}$ contain no point of $l$. Let $l^{\prime}$ denote a line perpendicular to $l$ and containing a point of $l \cap S_{L}$. Let $S^{\prime}$ denote any subset of $l \cap S_{L}$ contained in one of the open half planes bounded by $l^{\prime}$. If $(x, y)$ is any point of $R_{L} \cap l^{\prime}$, then

$$
H_{L}\left[(x, y), S^{\prime}, \Omega_{L}\right] \leq 1 / 2 .
$$

Proof. The theorem follows easily by (4.2), (4.3), and problem I.

As a corollary we have an upper bound of $\frac{1}{2}$ for problem II. Also, for problem III if we extend the lines whose harmonic measure is required to the right, we get by symmetry as in 
problem I, $\frac{1}{2}$ as an upper bound for the d.h.m. on the left dotted line. Similarly $\frac{1}{2}$ is an upper bound for the d.h.m. on the right dotted line.

For problem IV the situation is somewhat more complicated. We prove

Theorem 7.3: Let $\Omega$ denote the region: $x \geq 0, y \geq 0$, and let $\Omega_{L}$ denote the subset of $L[h, k]$ contained in $\Omega$. Let $I_{a}, I_{b}$ denote respectively the intervals $0<x<a, y=0$ and $0<y<b, x=0$, where the point $(a, b)$ belongs to $L[h, k]$. We have

where

$$
H_{L}\left[(x, b), I_{a} \cup I_{b}, \Omega_{L}\right] \leq \operatorname{Max}\left[\frac{1+w}{2} ; 1-\frac{(1-w) b}{2 y_{0}}\right]
$$

$$
w=(2 / \pi) \log \frac{1+\exp (-\pi / 2)}{1-\exp (-\pi / 2)}
$$

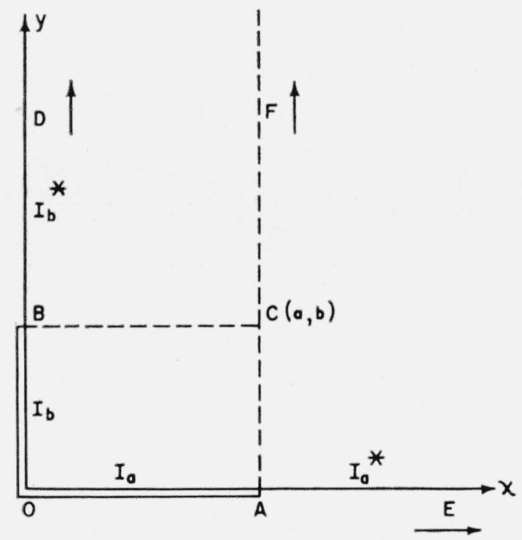

Figure 4.

Here $y_{0}$ is the smallest number not less than $a / 2 \alpha$ such that $y_{0} / k$ is an integer. The quantities $\alpha$ and $\sigma$ are defined by (3.12) and (2.3), respectively.

Proof. Let $I_{a}^{*}, I_{b}^{*}$ denote respectively the intervals $x \geq a, y=0$, and $y \geq b, x=0$. We first prove

Lemma 7.1. If $0 \leq x, 0 \leq y$, then

$$
H_{L}\left[(x, b), I_{b}^{*}, \Omega_{L}\right] \geq H_{L}\left[(x, b), I_{b}, \Omega_{L}\right],
$$

and

$$
H_{L}\left[(a, y), I_{a}^{*}, \Omega_{L}\right] \geq H_{L}\left[(a, y), I_{a}, \Omega_{L}\right]
$$

Proof: By theorem 7.2, we have

$$
H_{L}[(x, b), y \geq b \cap x=0, x \geq 0] \geq H_{L}[(x, b), y<b \cap x=0, x \geq 0] .
$$

Hence, we certainly have

$$
\begin{aligned}
H_{L}[(x, b),\{y \geq b \cap x=0\} \cup\{-b<y<0 \cap x=0\}, x \geq 0] & \\
& \geq H_{L}[(x, b),\{0<y<b \cap x=0\} \cup\{y \leq-b \cap x=0\}, x \geq 0] .
\end{aligned}
$$

But by a sign-changing reflection about the line $y=0$, we can show that the left member of the above expression minus the right member equals $H_{L}\left[(x, b), I_{b}^{*}, \Omega_{L}\right]-H_{L}\left[(x, b), I_{b}, \Omega_{L}\right] \geq 0$.

This proves (7.3). Evidently (7.4) can be proved by the same method.

From lemma 7.1, we conclude that

$$
H_{L}\left[(x, b), I_{a} \cup I_{b}, \Omega_{L}\right] \leq H_{L}\left[(x, b), I_{a}, \Omega_{L}\right]+(1 / 2) H_{L}\left[(x, b), 0<y \cap x=0, \Omega_{L}\right] .
$$


But by (7.4) we have

$$
(1 / 2) H_{L}\left[(a, y), 0<y \cap x=0, \Omega_{L}\right]+H_{L}\left[(a, y), I_{a}, \Omega_{L}\right] \leq 1 / 2 .
$$

Therefore, the d.h. function

$$
W(x, y)=H_{L}\left[(x, y), I_{a}, \Omega_{L}\right]+(1 / 2) H_{L}\left[(x, y), 0<y \cap x=0, \Omega_{L}\right]
$$

satisfies the conditions

$$
W(x, 0)=1, \quad(0<x<b) ; \quad W(0, y)=1 / 2, \quad(0<y) ; \quad W(a, y) \leq 1 / 2, \quad(0 \leq y) .
$$

Let $\Omega^{*}$ denote the region $0 \leq x \leq a \cap y \geq 0$, and let $\Omega_{L}^{*}$ denote the corresponding subset of $L[h, k]$. Evidently,

$$
W(x, y) \leq U_{1}(x, y)
$$

where

$$
\left.\begin{array}{l}
\text { (a) } U_{1}(x, y) \text { is d.h. and bounded in } R_{L}^{*} \\
\text { (b) } U_{1}(x, 0)=1, \quad(0<x<a) \\
\text { (c) } U_{1}(0, y)=U_{1}(a, y)=1 / 2, \quad(y \geq 0) .
\end{array}\right\}
$$

We now prove

Lemma 7.2. Let $U_{2}(x, y)$ be d.h. and bounded for $(x, y) \epsilon R_{L}^{*}$ and such that

$$
U_{2}(x, 0)=1, \quad(0<x<1) ; \quad U_{2}(0, y)=U_{2}(a, y)=0, \quad(y \geq 0) .
$$

We have

$$
U_{2}(x, c) \leq \operatorname{Max}\left[w ; 1-(1-w) c / y_{0}\right]
$$

Proof. Substituting in (3.5) and (3.6) and taking the limit as $b$ becomes infinite we get

$$
U_{2}(x, y)=\sum_{\substack{n=1 \\ n \text { odd }}}^{A}(2 / A) \cot (n \pi / 2 A) \sin (n \pi x / a) \exp (-m \pi y / a),
$$

where $A h=a$.

By lemma 3.2, we have $m \geq \alpha n$. Also, since $\cot [n \pi / 2 A] \leq 2 A / n \pi$ for $n \leq A$ we have

$$
U_{2}(x, y) \leq \sum_{\substack{n=1 \\ n \text { odd }}}^{A}(4 / \pi n) \exp (-\pi \alpha y n / a) \leq \sum_{\substack{n=1 \\ n \text { odd }}}^{\infty}(4 / \pi n) \exp (-\pi \alpha y n / a)=(2 / \pi) \log \frac{1+\exp (-\pi \alpha y / a)}{1-\exp (-\pi \alpha y / a)}
$$

Evidently if $y \geq a / 2 \alpha$, we have

$$
U_{2}(x, y) \leq w
$$

Now since $0<w<1$, the function

$$
U_{3}(x, y)=1-(1-w) y / y_{0}
$$

is $d . h$ in $R_{L}^{*}$ and is not less than $U_{2}(x, y)$ for $S_{L}^{*} \cap y \leq y_{0}$ and for $y=y_{0}$. Therefore, for $y \leq y_{0}$ we have

$$
U_{2}(x, y) \leq 1-(1-w) y / y_{0},
$$


and the lemma follows.

Now $U_{2}(x, y)=2 U_{1}(x, y)-1$. We also have by (7.5), (7.6), and (7.8).

$$
H_{L}\left[(x, b), I_{a} \cup I_{b}, \Omega_{L}\right] \leq W(x, b) \leq U_{1}(x, b),
$$

and the theorem now follows.

In applying the theorem to problem IV, we note that we can assume the value zero at the point $(0,0)$ without changing the d.h.m.

Thus, an error bound can be obtained for the case of two overlapping rectangles. The above methods can also be used to find error bounds for other regions of the type considered in section 2 .

\section{References}

[1] C. Carathéodory, Funktionentheorie, II (Verlag Birkhäuser Basel, 1950).

[2] L. Collatz, Bemerkungen zur Fehlerabschätzung für das Differenzenverfahren bei partiellen Differentialgleichungen, Z. angew. Math. u. Mech. 13, 56-57 (1933).

[3] E. Copson, Functions of a complex variable (Oxford Press, Cambridge 1935).

[4] S. Gerschgorin, Fehlerabschätzung für das Differenzenverfahren zur Lösung partieller Differentialgleichungen, Z. angew. Math. u. Mech. 10, 373-382 (1930).

[5] H. Heilbronn, On discrete harmonic functions, Proc. Cambridge Phil. Soc. 45, 194-206 (1949).

[6] D. Jackson, On the degree of convergence of Sturm-Liouville series, Trans. Am. Math. Soc. 15, 439-466 (1914).

[7] D. Jackson, The theory of approximation, Am. Math. Soc. Coll. Pub. XI (1930).

[8] D. Jackson, Fourier series and orthogonal polynomials, Mathematical Association of America Carus Monograph No. 6 (1941).

[9] J. Le Roux, Sur le problème de Dirichlet, J. Math. 10, 189-230 (1914).

[10] S. Mikeladze, Utber die numerische Lösung der Differentialgleichungen von Laplace und Poisson, Bul. acad. sci. U. S. S. R. (Ser. Math.) No. 2, 271-290 (1938).

[11] R. Nevanlinna, Eindeutige analytische Funktionen (Berlin, 1936).

[12] H. Phillips and N. Wiener, Nets and the Dirichlet problem, J. Math. Phys. 2, 105-124 (1923).

[13] P. C. Rosenbloom, On the difference equation method for solving the Dirichlet problem, Construction and applications of conformal maps, NBS AMS 18, 231 (1952).

[14] J. L. Walsh and D. Young, On the accuracy of the numerical solution of the Dirichlet problem by finite differences, Bul. Am. Math. Soc. 5\%, Abstract 500t, p. 478 (1951).

[15] W. Wasow, On the truncation error in the solution of Laplace's equation by finite differences, J. Research NBS 48, 345-348 (1952) RP2321.

Washington, January 6, 1953. 